\title{
基于偶氮桥连的新型双铁羰基配合物: $\mathrm{Fe}_{2}\left(\mathrm{~N}_{2} \mathrm{C}_{5} \mathrm{H}_{10}\right)(\mathrm{CO})_{6-x}\left(\mathrm{PR}_{3}\right)_{x}$ 的 合成及衍生
}

\author{
李苒徐文元赵巾钦余釒全文光* 佟振合 \\ (山东大学化学与化工学院 济南 250100)
}

\begin{abstract}
摘要 具有五元环结构的偶氮化合物 4,4-二甲基-4,5-二氢-3H-吡咯 $\left(\mathrm{N}_{2} \mathrm{C}_{5} \mathrm{H}_{10}\right)$, 与 $\mathrm{Fe}_{3}(\mathrm{CO})_{12}$ 在甲苯中加热回流反应, 生 成双铁六羰基配合物 $\mathrm{Fe}_{2}\left(\mathrm{~N}_{2} \mathrm{C}_{5} \mathrm{H}_{10}\right)(\mathrm{CO})_{6}(\mathbf{1})$. 反应中 $\mathrm{N}=\mathrm{N}$ 双键被还原, 配体以 $\left(\mathrm{N}_{2} \mathrm{C}_{5} \mathrm{H}_{10}\right)^{2-}$ 的形式与 $\mathrm{Fe}^{\mathrm{I}} \mathrm{Fe}^{\mathrm{I}}$ 配位, 形成 具有蝶形结构的 $34 \mathrm{e}^{-}$化合物. 研究了在脱羰基试剂 $\mathrm{Me}_{3} \mathrm{NO}$ 存在条件下, 1 和单齿膦配体 $\mathrm{PR}_{3}$ 反应生成 $\mathrm{Fe}_{2}\left(\mathrm{~N}_{2} \mathrm{C}_{5} \mathrm{H}_{10}\right)-$ $(\mathrm{CO})_{5}\left(\mathrm{PR}_{3}\right)\left(\mathrm{PR}_{3}=\mathrm{PPh}_{3}, \mathbf{2 a} ; \mathrm{PCy}_{3}, \mathbf{2 b}\right)$ 单取代配合物. 光照条件下, 化合物 $\mathbf{1}$ 中的 $\mathrm{CO}$ 配体还可以被双齿膦配体 dppe $\left[\mathrm{dppe}=1,2-\mathrm{C}_{2} \mathrm{H}_{4}\left(\mathrm{PPh}_{2}\right)_{2}\right]$ 和 $\mathrm{dppbz}\left[\mathrm{dppbz}=1,2-\mathrm{C}_{6} \mathrm{H}_{4}\left(\mathrm{PPh}_{2}\right)_{2}\right]$ 取代, 生成产物的类型和膦配体的夹角相关. 与夹角较大的 dppe 反应, 生成桥连产物 $\mathrm{Fe}_{2}\left(\mathrm{~N}_{2} \mathrm{C}_{5} \mathrm{H}_{10}\right)(\mathrm{CO})_{4}\left(\mu\right.$-dppe) (3a); 而与刚性较大的 dppbz 反应时, $\mathrm{Fe}_{2}(\mathrm{NR})_{2}$ 的蝶形结构打开呈 四元环; 其中一个 $\mathrm{Fe}$ 上的 $\mathrm{CO}$ 被取代, $\mathrm{dppbz}$ 与该 $\mathrm{Fe}$ 中心螯合, 生成具有桥连 $\mathrm{CO}$ 的化合物 $\mathrm{Fe}_{2}\left(\mathrm{~N}_{2} \mathrm{C}_{5} \mathrm{H}_{10}\right)(\mu-\mathrm{CO})(\mathrm{CO})_{4}\left(\kappa^{2}-\right.$ dppbz) (3b). 合成具有 $\mathrm{Fe}^{\mathrm{I}}-\mathrm{CO}-\mathrm{Fe}^{\mathrm{I}}$ 结构的羰基化合物, 一直是模拟 $[\mathrm{FeFe}]$ 氢化酶活性中心还原态结构 $\mathrm{Fe}_{2}(\mathrm{SR})_{2}(\mu-\mathrm{CO})-$ $(\mathrm{CO})_{5-x} \mathrm{~L}_{x}$ 的重要挑战. 该类 $\mathrm{Fe}_{2}(\mathrm{NR})_{2}(\mathrm{CO})_{6-x}\left(\mathrm{PR}_{3}\right)_{x}$ 化合物的合成, 能为探索模拟 $[\mathrm{FeFe}]$ 氢化酶活性中心结构提供新的途 径和思路. 以上化合物均通过核磁 $\left[{ }^{31} \mathrm{P}\left({ }^{1} \mathrm{H}\right) \mathrm{NMR}\right]$ 、红外光谱(IR)、元素分析及 $\mathrm{X}$ 射线单晶结构衍射等表征.
\end{abstract} 关键词 $[\mathrm{FeFe}]$ 氢化酶; 双铁羰基配合物; 膦配体; 旋转构型; 桥联 $\mathrm{CO}$ 配体

\section{Azo-bridged New Diiron Carbonyl Complex: Synthesis of $\mathrm{Fe}_{2}(\mathrm{NR})_{2}(\mathrm{CO})_{6-x}\left(\mathrm{PR}_{3}\right)_{x}$ and the Derivatives}

\author{
$\mathrm{Li}$, Ran $\quad \mathrm{Xu}$, Wenyuan Zhao, Jinqin Yu, Xin Wang, Wenguang* Tung, Chen-Ho \\ (Department of Chemistry \& Chemical Engineering, Shandong University, Jinan 250100, China)
}

Abstract Heating the toluene solution of 4,4-dimethyl-4,5-dihydro-3H-pyrazole $\left(\mathrm{N}_{2} \mathrm{C}_{5} \mathrm{H}_{10}\right)$ and $\mathrm{Fe}_{3}(\mathrm{CO})_{12}$ at reflux for $1 \mathrm{~h}$ produces diiron hexacarbonyls $\mathrm{Fe}_{2}\left(\mathrm{~N}_{2} \mathrm{C}_{5} \mathrm{H}_{10}\right)(\mathrm{CO})_{6}\left(1, v_{\mathrm{CO}}\left(\mathrm{CH}_{2} \mathrm{Cl}_{2}\right): 2069,2022,1986 \mathrm{~cm}^{-1}\right)$. Compound 1 exhibits $34 \mathrm{e}^{-}$ configuration, in which $\left(\mathrm{N}_{2} \mathrm{C}_{5} \mathrm{H}_{10}\right)^{2-}$ coordinates to diiron $\left(\mathrm{Fe}^{\mathrm{I}} \mathrm{Fe}^{\mathrm{I}}\right)$ centers featuring a butterfly structure. To a solution of $\mathbf{1}$ in toluene was added one equiv. of decarbonyl agent $\mathrm{Me}_{3} \mathrm{NO}$ in $\mathrm{MeCN}$, and the mixture was stirred at room temperature for 20 min. Then, one equiv. of monophosphine was added. After $3 \mathrm{~h}$, the solvent was removed and the residue was extracted into 5 mL $\mathrm{CH}_{2} \mathrm{Cl}_{2}$. The product $\mathrm{Fe}_{2}\left(\mathrm{~N}_{2} \mathrm{C}_{5} \mathrm{H}_{10}\right)(\mathrm{CO})_{5}\left(\mathrm{PR}_{3}\right)\left(\mathrm{PR}_{3}=\mathrm{PPh}_{3}, \mathbf{2 a} ; \mathrm{PCy}_{3}, \mathbf{2 b}\right)$ was obtained as brown crystals by allowing a pentane layer to diffuse into the $\mathrm{CH}_{2} \mathrm{Cl}_{2}$ solution at $-20{ }^{\circ} \mathrm{C}$. ${ }^{31} \mathrm{P}$ NMR spectra exhibit a singlet at $\delta 67$ for $2 \mathbf{a}$ and $\delta 70$ for $\mathbf{2 b}$ in $\mathrm{CH}_{2} \mathrm{Cl}_{2}$, respectively. In IR spectra, the $v_{\mathrm{CO}}$ bands for $2 \mathrm{a}$ were displayed at $2032,1968,1952,1907 \mathrm{~cm}^{-1}$, which are compared to 2024, 1959, 1937, $1893 \mathrm{~cm}^{-1}$ for $\mathbf{2 b}$. Photolysis the toluene solution of $\mathbf{1}$ in the presence of chelating diphosphine ligands such as dppe [dppe $\left.=1,2-\mathrm{C}_{2} \mathrm{H}_{4}\left(\mathrm{PPh}_{2}\right)_{2}\right]$ and dppbz [dppbz=1,2- $\left.\mathrm{C}_{6} \mathrm{H}_{4}\left(\mathrm{PPh}_{2}\right)_{2}\right]$ affords diiron diphosphine carbonyl compounds. For dppe, the product was $\mathrm{Fe}_{2}\left(\mathrm{~N}_{2} \mathrm{C}_{5} \mathrm{H}_{10}\right)(\mathrm{CO}){ }_{4}\left(\mu\right.$-dppe) $\left(3 \mathrm{a},{ }^{31} \mathrm{P}\right.$ NMR $\left(\mathrm{CD}_{2} \mathrm{Cl}_{2}\right): \delta$ 95, FT-IR $\left(\mathrm{CH}_{2} \mathrm{Cl}_{2}, v_{\mathrm{CO}}\right)$ : $1984,1940,1925$ and $\left.1900 \mathrm{~cm}^{-1}\right)$, in which dppe is bridging the two iron centers. For more rigid diphosphine ligand dppbz, $\mathrm{X}$-ray crystallographic analysis reveals the structure of $\mathrm{Fe}_{2}\left(\mathrm{~N}_{2} \mathrm{C}_{5} \mathrm{H}_{10}\right)(\mu-\mathrm{CO})(\mathrm{CO})_{4}(\mathrm{dppbz})\left[3 \mathbf{b},{ }^{31} \mathrm{P} \mathrm{NMR}\left(\mathrm{CD}_{2} \mathrm{Cl}_{2}\right): \delta 93\right]$. In 3b, $\left(\mathrm{N}_{2} \mathrm{C}_{5} \mathrm{H}_{10}\right)^{2-}$ coordinates to diiron centers in a planar mode, and dppbz chelates at one $\mathrm{Fe}$ site by the replacement of one $\mathrm{CO}$ ligand. Compound $\mathbf{3 b}$ features a Fe-CO-Fe rotated structure with a bridging $\mathrm{CO}$ ligand between the two Fe centers. The $v_{\mathrm{CO}}$ bands for $\mathbf{3 b}$ were displayed at 1990, 1947, 1919, $1895 \mathrm{~cm}^{-1}$. With such a rotated structure, compound $\mathbf{3 b}$ provides a new approach for synthetic models of $\mathrm{H}_{\text {red }}$ state of $[\mathrm{FeFe}]-\mathrm{H}_{2}$ ase. The CCDC number for 1, 2a, 2b, 3a and 3b are 1494954, 1494955, 1494956, 1494966 and 1494957. All the compounds were well characterized by NMR, IR spectroscopy and elemental analysis.

Keywords $[\mathrm{FeFe}]$ hydrogenase; diiron carbonyls; phosphine ligands; rotated structure; bridging CO ligand

\footnotetext{
*E-mail:wwg@sdu.edu.cn

Received July 27, 2016; published October 11, 2016
}

Supporting information for this article is available free of charge via the Internet at http://sioc-journal.cn.

Project supported by the National Natural Science Foundation of China (Nos. 21402107, 91427303), the financial support from the "1000 Youth Talents Plan" and National Undergraduate Training Programs for Innovation and Entrepreneurship (No. 201510422032).

项目受国家自然科学基金(Nos. 21402107, 91427303), “青年千人” 计划 和 “国家级大学生创新创业训练计划(No. 201510422032)”资助. 


\section{1 引言}

$[\mathrm{FeFe}]$-氢化酶能够可逆地催化质子 $\left(\mathrm{H}^{+}\right)$, 电子 $\left(\mathrm{e}^{-}\right)$ 和氢气 $\left(\mathrm{H}_{2}\right)$ 之间的转换反应: $2 \mathrm{H}^{+}+\mathrm{e}^{-} \leftrightarrow \mathrm{H}_{2}$, 特别是其高 效的产 $\mathrm{H}_{2}$ 效率 $\left(\mathrm{TOF} \approx 6000 \sim 9000 \mathrm{~s}^{-1}\right.$ ), 引起了化学家 们的研究兴趣 ${ }^{[1,2]}$. [ FeFe]-氢化酶活性中心的结构如图 1a 所示 ${ }^{[3]}$, 主要包含蝶形结构的 $\mathrm{Fe}_{2} \mathrm{~S}_{2}$ 骨架, 连接在 $\mathrm{Fe}$ 上的 3 个 $\mathrm{CO} 、 2$ 个 $\mathrm{CN}^{-}$配体及 $\left[\mathrm{Fe}_{4} \mathrm{~S}_{4}\right]$ 簇. 其中, $\mathrm{Fe}_{\mathrm{p}}$ 上 的 $\left[\mathrm{Fe}_{4} \mathrm{~S}_{4}\right.$ ] 簇在催化过程中起着转移 $\mathrm{e}^{-}$的作用 ${ }^{[4]}$; 两个 $\mathrm{Fe}$ 共享一个桥连 $\mathrm{CO}$ 配体( $\mu$ - $\mathrm{CO}$ ), 催化活性位点位于 $\mathrm{Fe}_{\mathrm{d}}$ 的末端 ${ }^{[5]}$, 与 $\mu$ - $\mathrm{CO}$ 处于反式位置. 这种具有 $\mathrm{Fe}-\mathrm{CO}-\mathrm{Fe}$ 的结构称为 “旋转” 构型(“rotated” structure).

(a)
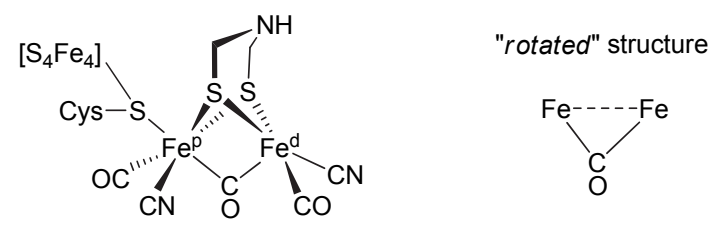

(b)

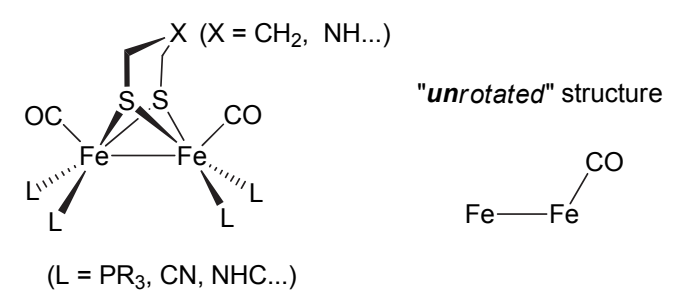

图 1 (a) [FeFe]-氢化酶活性中心的结构; (b) 模型化合物示意图 Figure 1 Structures for the active site of $[\mathrm{FeFe}]-\mathrm{H}_{2}$ ase (a) and the synthetic models (b)

由于 $\mathrm{CN}^{-}$配体的 $\mathrm{N}$ 可以和蛋白中的氨基酸残基形 成 $\mathrm{Fe}_{\mathrm{d}} \mathrm{CN} \cdots \mathrm{H}_{3} \mathrm{~N}-\mathrm{Lys}_{358}$ 氢键作用 ${ }^{[6]}$, 尽管在催化过程中 $\mathrm{Fe}$ 的化合价态不断变化, 但活性中心始终维持 “旋转” 的构型, 使得催化 $\mathrm{H}^{+}$还原反应能够高效进行(通常情况 下, 金属中心价态变化, 会导致构型翻转, 从而降低中 间体的能量或活性). 通过顺磁共振、红外、拉曼等对蛋 白中提取出的氢化酶进行表征, 发现按照 $\mathrm{FeFe}$ 中心的 化合价态可分为氧化态 $\left(\mathrm{H}_{\mathrm{ox}}\right)$ 和还原态 $\left(\mathrm{H}_{\mathrm{red}}\right)$ 两种. 其中, 氧化态为 $\mathrm{Fe}^{\mathrm{II}} \mathrm{Fe}$ 价态; 而还原态可能为 $\mathrm{Fe}^{\mathrm{I}} \mathrm{Fe}^{\mathrm{I}}$ 或质子化 的 $\mathrm{HFe}^{\mathrm{II}} \mathrm{Fe}^{\mathrm{II}[7 \sim 9]}$.

为了模拟 $[\mathrm{FeFe}]$-氢化酶活性中心的结构和功能, 化 学家们致力于合成模型化合物 $\mathrm{Fe}_{2}(\mathrm{SR})_{2}(\mathrm{CO})_{6-\mathrm{x}} \mathrm{L}_{x}(\mathrm{~L}=$ $\mathrm{PR}_{3}{ }^{[10,11]}, \mathrm{CN}^{-[12]}, \mathrm{NHC}^{[13]}$ 等配体, 图 $\left.1 \mathrm{~b}\right)$, 研究其催化质 子还原的机制, 理解 “结构-功能”之间的关系, 寻求发

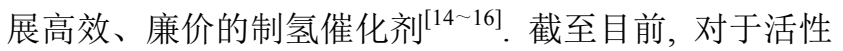
中心氧化态的模拟已取得了很大的进展. Darensbourg ${ }^{[17]}$ 和 Rauchfuss ${ }^{[18]}$ 等首次报道了氧化态模型化合物 $\left[\mathrm{Fe}_{2}(\mathrm{~S}-\right.$ $\left.\left.\mathrm{C}_{3} \mathrm{H}_{6} \mathrm{~S}\right)(\mathrm{CO})_{4}(\mathrm{IMes})\left(\mathrm{PMe}_{3}\right)\right]^{+}$和 $\left[\mathrm{Fe}_{2}\left(\mathrm{SC}_{2} \mathrm{H}_{4} \mathrm{~S}\right)(\mathrm{CO})_{4}(\mathrm{dppv})-\right.$ $\left.\left(\mathrm{PMe}_{3}\right)\right]^{+}$具有完美的 “旋转” 构型. 随后, 很多研究发现 这类 $\mathrm{Fe}^{\mathrm{II}} \mathrm{Fe}^{\mathrm{I}}$ 模型化合物被氧化后, 能实现 $\mathrm{H}_{2}$ 的异裂, 并
生成含有桥连 “负氢” $\left(\mathrm{H}^{-}\right)$的产物 $\left[\mathrm{Fe}^{\mathrm{II}}-\mathrm{H}-\mathrm{Fe}^{\mathrm{II}}\right]^{+[19]}$.

文献报道的 $\mathrm{Fe}_{2}(\mathrm{SR})_{2}(\mathrm{CO})_{6-x} \mathrm{~L}_{x}$ 模型化合物, 通常不 具有 $\mathrm{Fe}-\mathrm{CO}-\mathrm{Fe}$ 的 “旋转” 构型, 而是以最低能量状态的 $\mathrm{Fe}-\mathrm{Fe}-\mathrm{CO}$ 构型存在. 含有双齿膦配体 (P-P)的不对称取 代化合物 $\mathrm{Fe}_{2}(\mathrm{SR})_{2}(\mathrm{CO})_{4}(\mathrm{P}-\mathrm{P})$, 虽然不具有 “旋转” 构型, 但在 $-40{ }^{\circ} \mathrm{C}$ 结合 $\mathrm{H}^{+}$时, $\mathrm{Fe}^{\mathrm{I}}-\mathrm{Fe}^{\mathrm{I}}-\mathrm{CO}$ 生成具有 “旋转” 构 型的 $\mathrm{Fe}^{\mathrm{II}}-\mathrm{CO}-\mathrm{Fe}^{\mathrm{II}}-\mathrm{H}$. 该类型产物为动力学产物, 构型不 稳定, 室温时异构化为 $\mathrm{Fe}^{\mathrm{II}}-\mathrm{H}-\mathrm{Fe} \mathrm{e}^{\mathrm{II}}-\mathrm{CO}$ (图 2) ${ }^{[20]}$. 研究发 现，电化学条件下 $\mathrm{Fe}^{\mathrm{II}}-\mathrm{CO}-\mathrm{Fe}^{\mathrm{II}}-\mathrm{H}$ 催化 $\mathrm{H}^{+}$还原制 $\mathrm{H}_{2}$ 的过 电位低于 $\mathrm{Fe}^{\mathrm{II}}-\mathrm{H}-\mathrm{Fe}^{\mathrm{II}}-\mathrm{CO}^{[21]}$.

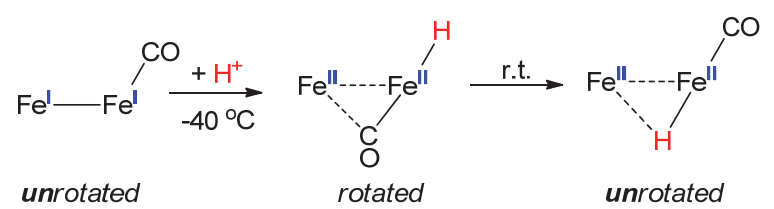

图 2 不对称取代化合物 $\mathrm{Fe}_{2}(\mathrm{SR})_{2}(\mathrm{CO})_{4}(\mathrm{P}-\mathrm{P})$ 和酸 $\left(\mathrm{H}^{+}\right)$反应的构型变化 示意图

Figure 2 Protonation of the unsymmetrically substituted $\mathrm{Fe}_{2}(\mathrm{SR})_{2}(\mathrm{CO})_{4}$ (P-P) compounds in which P-P is chelating diphosphine ligand

合成具有 “旋转” 构型的 $\mathrm{Fe}^{\mathrm{I}}-\mathrm{CO}-\mathrm{Fe}^{\mathrm{I}}$ 化合物, 一直 是模拟 [ FeFe]-氢化酶活性中心还原态结构的难点所 在 ${ }^{[22]}$. 最近, 文献报道 “旋转” 构型的 $\mathrm{Fe}^{\mathrm{I}}-\mathrm{CO}-\mathrm{Fe} \mathrm{F}^{\mathrm{I}}$ 化合 物的合成可以通过增大空间位阻和不对称取代的策略 初步实现 ${ }^{[23,24]}$. 例如, 化合物 $(\mathrm{CO})_{3} \mathrm{Fe}\left(\mathrm{SCH}_{2} \mathrm{CMe}_{2} \mathrm{CH}_{2} \mathrm{~S}\right)-$ $\mathrm{Fe}(\mathrm{dppv})(\mathrm{CO})\left(\mathrm{dppv}=c i s-1,2-\mathrm{Ph}_{2} \mathrm{PC}_{2} \mathrm{H}_{2} \mathrm{PPh}_{2}\right)$ 中 ${ }^{[23]}$, 在桥 头引入双甲基取代基, 结合在其中一个 $\mathrm{Fe}$ 上螯合的双 齿膦配体, 可以分离得到 $\mathrm{Fe}^{\mathrm{I}}-\mathrm{CO}-\mathrm{Fe}^{\mathrm{I}}(13 \%)$ 和 $\mathrm{Fe}^{\mathrm{I}}-\mathrm{Fe}^{\mathrm{I}}-\mathrm{CO}$ (87\%)两种异构体. 为了合成稳定构型的 $\mathrm{Fe}^{\mathrm{I}}-\mathrm{CO}-\mathrm{Fe}^{\mathrm{I}}$ 化 合物, 化学家们还探索将 $\mathrm{Fe}_{2} \mathrm{~S}_{2}$ 簇拓展为 $\mathrm{Fe}_{2} \mathrm{X}_{2}$, 如 $\mathrm{Fe}_{2}(\mathrm{SeR})_{2}(\mathrm{CO})_{6-x} \mathrm{~L}_{x}{ }^{[25]}, \mathrm{Fe}_{2}\left(\mathrm{PR}_{2}\right)_{2}(\mathrm{CO})_{6-\mathrm{x}} \mathrm{L}_{x}{ }^{[26]}$ 等.

相比于 $\mathrm{Fe}_{2} \mathrm{Se}_{2}$ 簇和 $\mathrm{Fe}_{2} \mathrm{P}_{2}$ 簇, 文献关于 $\mathrm{Fe}_{2} \mathrm{~N}_{2}$ 簇类化 合物的报道极少. 2007 年, Rauchfuss 课题组 ${ }^{[27]}$ 报道了 以噌啉 $\left(\mathrm{N}_{2} \mathrm{C}_{12} \mathrm{H}_{8}, \mathrm{BC}\right)$ 为配体的 $\mathrm{Fe}_{2} \mathrm{~N}_{2}$ 簇 $\mathrm{Fe}_{2}(\mathrm{BC})(\mathrm{CO})_{6}$, 研究了其 $\mathrm{CO}$ 配体的取代反应, 合成了对称取代结构的 $\mathrm{Fe}^{\mathrm{I}}-\mathrm{Fe}^{\mathrm{I}}-\mathrm{CO}$ 类化合物 $\left[\mathrm{Fe}(\mathrm{CO})_{2}\left(\mathrm{PMe}_{3}\right)\right]_{2}(\mathrm{BC})$. 迄今为止, 有关对其它 $\mathrm{Fe}_{2} \mathrm{~N}_{2}$ 簇进行非对称取代反应的研究还未见 报道. 本文利用五元环偶氮化合物 4,4-二甲基-4,5-二氢$3 H$-吡咯为配体, 合成了 $\mathrm{Fe}_{2}\left(\mathrm{~N}_{2} \mathrm{C}_{5} \mathrm{H}_{10}\right)(\mathrm{CO})_{6}$ (化合物 1); 研究了单、双齿膦配体对其 $\mathrm{CO}$ 配体的取代反应, 合成 了一系列膦配体衍生物 $\mathrm{Fe}_{2}\left(\mathrm{~N}_{2} \mathrm{C}_{5} \mathrm{H}_{10}\right)(\mathrm{CO})_{6-x}\left(\mathrm{PR}_{3}\right)_{x}$. 特别 重要的是, 1 和 $\mathrm{dppbz}$ 反应, 生成具有 “旋转” 构型的 $\mathrm{Fe}^{\mathrm{I}}-\mathrm{CO}-\mathrm{Fe}$ 羰基化合物 $\mathbf{3 b}$.

\section{2 结果与讨论}

\section{1 化合物 $\mathrm{Fe}_{2}\left(\mathrm{~N}_{2} \mathrm{C}_{5} \mathrm{H}_{10}\right)(\mathrm{CO})_{6} 1$ 的合成及表征}

如 Eq. 1 所示, 将溶解有 4,4-二甲基-4,5-二氢-3H吡咯配体和 $\mathrm{Fe}_{3}(\mathrm{CO})_{12}$ 的甲苯溶液, 在 $\mathrm{N}_{2}$ 保护条件下加 热回流. 溶液颜色逐渐由墨绿色变为棕色, 利用傅利叶 
红外光谱跟踪反应进程, 反应约 $2 \mathrm{~h}$ 完成. 经减压蒸出 溶剂, 柱层析(乙酸乙酯/石油醚 $=1 / 10$ ) 分离后, 得到橙 红色固体产物 1, 分离产率 $68 \%$.

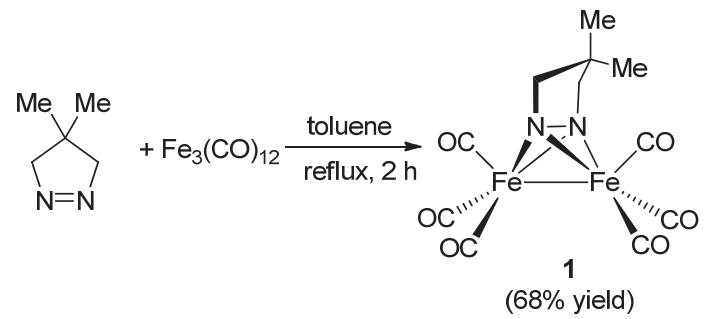

化合物的 1 的 ${ }^{1} \mathrm{H}$ NMR 显示为两组单峰, 化学位移 分别位于 $\delta 3.41(4 \mathrm{H})$ 和 $\delta 1.32(6 \mathrm{H})$, 对应于 $\mathrm{CH}_{2}$ 和 $\mathrm{CH}_{3}$ 的核磁共振吸收峰. IR 谱图显示, 1 在 $1900 \sim 2100 \mathrm{~cm}^{-1}$ 范围内有三个 $\mathrm{CO}$ 共振吸收带 $v_{\mathrm{CO}}$ : 2069, 2022, 1986 $\mathrm{cm}^{-1}$. 与化合物 $\mathrm{Fe}_{2}\left(\mathrm{SCH}_{2} \mathrm{CMe}_{2} \mathrm{CH}_{2} \mathrm{~S}\right)(\mathrm{CO})_{6}$ 的 $\mathrm{CO}$ 吸收 带 $\left(2073,2032,2003 \mathrm{~cm}^{-1}\right)^{[23]}$ 相比, 1 中 $\Delta v_{\mathrm{CO}}$ 向低波数移 动约 $10 \mathrm{~cm}^{-1}$, 表明该类型 $\mathrm{Fe}_{2}(\mathrm{NR})_{2}(\mathrm{CO})_{6}$ 化合物的 $\mathrm{Fe}^{\mathrm{I}} \mathrm{Fe}^{\mathrm{I}}$ 中心的电子云密度略强于化合物 $\mathrm{Fe}_{2}\left(\mathrm{SCH}_{2} \mathrm{CMe}_{2}-\right.$ $\left.\mathrm{CH}_{2} \mathrm{~S}\right)(\mathrm{CO})_{6}$.

我们通过 $X$ 射线单晶衍射分析确认了化合物 1 的结 构, 如图 3 所示. $\mathrm{N}=\mathrm{N}$ 双键打开呈 $(\mathrm{N}-\mathrm{N})^{2-}$ 阴离子形式, 分别与两个 $\mathrm{Fe}$ 配位, 形成蝶形的 $\mathrm{Fe}_{2} \mathrm{~N}_{2}$ 骨架结构. 其中 $\mathrm{Fe}-\mathrm{Fe}$ 键长为 $2.510(2) \AA$, 与 $\mathrm{Fe}_{2}\left(\mathrm{SC}_{3} \mathrm{H}_{6} \mathrm{~S}\right)(\mathrm{CO})_{6}$ 中的 $\mathrm{Fe}$ 一 $\mathrm{Fe}$ 键长相等 ${ }^{[12 \mathrm{a}]}$. 值得注意的是, 由于 $\mathrm{N}_{2} \mathrm{C}_{5} \mathrm{H}_{10}{ }^{2-}$ 配 体还处于五元环结构中, 张力较大, 使得 $\mathrm{N}-\mathrm{Fe}-\mathrm{N}$ 的夹 角(分别为 $49^{\circ}$ 和 $43^{\circ}$ ) 远远小于 $\mathrm{Fe}_{2}\left(\mathrm{SC}_{3} \mathrm{H}_{6} \mathrm{~S}\right)(\mathrm{CO})_{6}$ 中 $\mathrm{S}-\mathrm{Fe}-\mathrm{S}$ 的夹角 $85.2^{\circ}$. 从红外光谱和晶体数据分析可知, 化合物 1 保持着 $\mathrm{Fe}_{2}(\mathrm{SR})_{2}(\mathrm{CO})_{6}$ 类化合物的蝶形结构, 且 $\mathrm{Fe}(\mathrm{I}) \mathrm{Fe}(\mathrm{I})$ 中心有着相似的电子云密度.

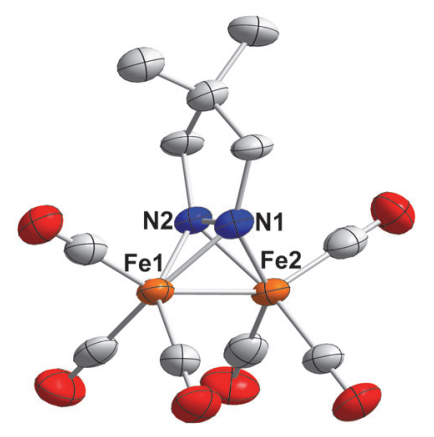

图 3 化合物 $\mathbf{1}$ 的晶体结构

Figure 3 Molecular structure (50\% probability thermal ellipsoids) of compound 1

\section{2 单齿磷配体取代产物 $\mathrm{Fe}_{2}\left(\mathrm{~N}_{2} \mathrm{C}_{5} \mathrm{H}_{10}\right)(\mathrm{CO})_{5}\left(\mathrm{PR}_{3}\right) \mathbf{2 a}$ 和 $2 b$ 的合成及表征}

$\mathrm{Fe}_{2}(\mathrm{SR})_{2}(\mathrm{CO})_{6}$ 模型化合物中的 $\mathrm{CO}$ 配体，可以被膦 配体取代, 以提高 $\mathrm{FeFe}$ 中心的电子云密度. 我们初步探 索了 $\mathrm{PPh}_{3}$ 和 $\mathrm{PCy}_{3}$ 与化合物 1 的 $\mathrm{CO}$ 取代反应. 如 Eq. 2 所示, 向溶有 1 的甲苯溶液中, 加入脱羰基试剂 $\mathrm{Me}_{3} \mathrm{NO}$
的乙腈溶液，反应 $20 \mathrm{~min}$ 后，再加入 $\mathrm{PPh}_{3}$ (或 $\mathrm{PCy}_{3}$ ). 室 温条件下搅拌 $3 \mathrm{~h}$, 反应基本完成. 产物 $\mathbf{2 a}$ 和 $\mathbf{2 b}$ 的分离 产率分别为 $81 \%$ 和 $73 \%$.

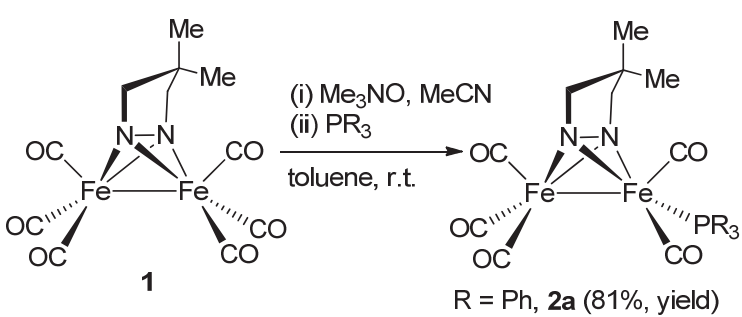

Cy, $\mathbf{2 b}(73 \%$, yield $)$

化合物 $\mathbf{2 a}$ 和 $\mathbf{2 b}$ 的 ${ }^{31} \mathrm{P}$ NMR 分别在 $\delta 67$ 和 $\delta 70$ 处 呈现一个单峰; ${ }^{1} \mathrm{H} \mathrm{NMR}$ 上与 $\mathrm{N}$ 相连的 $\mathrm{CH}_{2}$ 的化学位移 与化合物 1 相比没有明显移动, 但呈现出宽峰. 化合物 1 中的 $\mathrm{CO}$ 配体被膦配体取代后, $\mathrm{Fe}$ 核的电子云密度增 强，金属中心对 $\mathrm{CO}$ 有更强的反馈作用，表现为红外光 谱上 CO 的共振吸收带向低波数移动. 如图 4 所示, $\mathbf{2 a}$ 的红外光谱在 $2032,1968,1952,1907 \mathrm{~cm}^{-1}$ 呈现四个 CO 吸收峰, $\Delta v_{\mathrm{CO}}$ 向低波数移动了约 $50 \mathrm{~cm}^{-1} . \mathbf{2 b}$ 的红外光谱 轮廓和 $2 \mathbf{a}$ 相似, 其 $v_{\mathrm{CO}}$ 位置分别在 2024，1959，1937, $1893 \mathrm{~cm}^{-1}$.

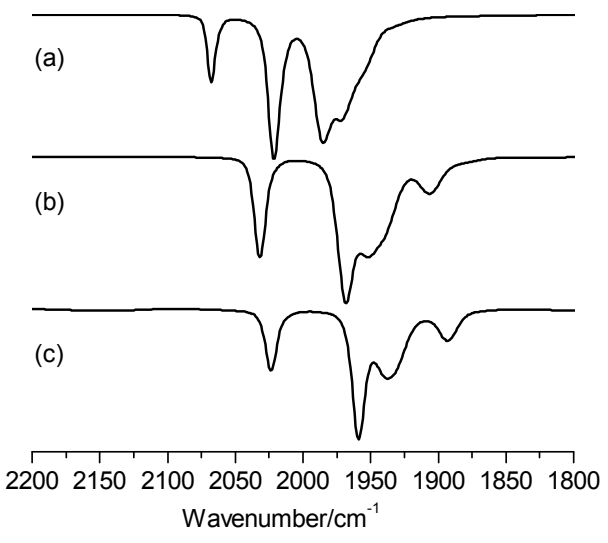

图 4 化合物 $\mathbf{1}(\mathrm{a}), \mathbf{2 a}(\mathrm{b})$ 和 $\mathbf{2 b}(\mathrm{c})$ 在 $v_{\mathrm{CO}}$ 的红外光谱图 Figure 4 IR spectra of $\mathbf{1}$ (a), 2a (b) and $\mathbf{2 b}$ (c) in $v_{\mathrm{CO}}$ region

化合物 $\mathbf{2 a}$ 和 $\mathbf{2 b}$ 的晶体结构如图 5 所示, 膦配体处 于其中一个 $\mathrm{Fe}$ 的底部(basal position), 分子保持了 $\mathrm{Fe}_{2} \mathrm{~N}_{2}$ 蝶形结构. $2 \mathrm{a}$ 和 $2 \mathrm{~b}$ 的 $\mathrm{Fe}-\mathrm{Fe}$ 键长分别为 2.5710(6) $\AA$ 和 2.6017(6) A, 见表 1. 随着化合物 1 中的 CO 被单齿膦配 体取代, $\mathrm{Fe}-\mathrm{Fe}$ 键长增加. 膦配体的供电子能力越强, 其键长越长. 另外, $\mathrm{N}-\mathrm{Fe}-\mathrm{N}$ 的键角也有稍许改变, 变 化范围在 $1^{\circ} \sim 2^{\circ}$ 之间. 由此可见, 对于这种单齿膦配体 的不对称取代反应，对分子的构型影响很小. 因此，我 们继续探索对化合物 1 进行双齿膦配体 P-P 的不对称取 代反应. 


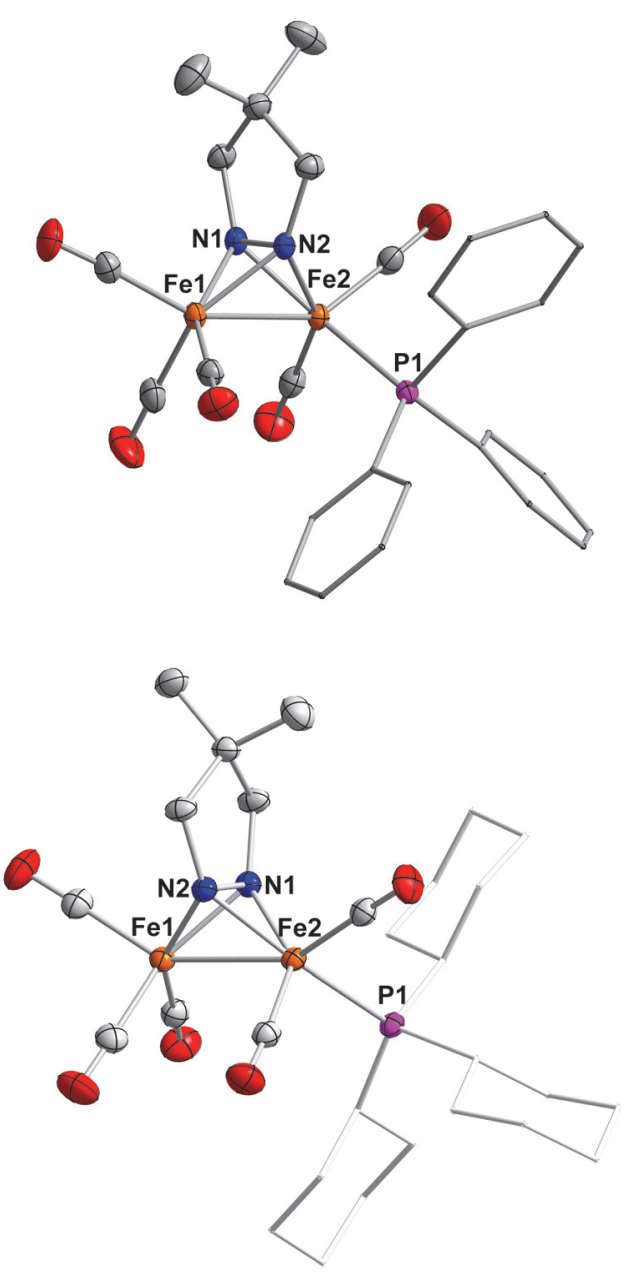

图 5 化合物 $\mathbf{2 a}$ 和 $\mathbf{2 b}$ 的晶体结构

Figure 5 Molecular structures (50\% probability thermal ellipsoids) of compound $\mathbf{2 a}$ and $\mathbf{2 b}$. For clarity, the phenyl groups, cyclohexyl groups and hydrogen atoms are omitted

表 1 化合物 $1,2 \mathrm{a}, 2 \mathrm{~b}, 3 \mathrm{a}$ 和 $3 \mathrm{~b}$ 的部分键长 $(\AA)$ 与键角 $\left({ }^{\circ}\right)$ 数据

Table 1 Selected bond lengths $(\AA)$ and angles $\left({ }^{\circ}\right)$ for 1, 2a, 2b, 3a and $3 \mathbf{b}$

\begin{tabular}{lccccc}
\hline & $\mathbf{1}$ & $\mathbf{2 a}$ & $\mathbf{2 b}$ & $\mathbf{3 a}$ & $\mathbf{3 b}$ \\
\hline $\mathrm{Fe}(1)-\mathrm{Fe}(2)$ & $2.510(2)$ & $2.5710(6)$ & $2.6017(6)$ & $2.558(1)$ & $2.5881(6)$ \\
$\mathrm{N}(1)-\mathrm{N}(2)$ & $1.40(1)$ & $1.398(2)$ & $1.403(1)$ & $1.404(5)$ & $1.280(3)$ \\
$\mathrm{Fe}(1)-\mathrm{N}(1)$ & $1.893(8)$ & $1.904(1)$ & $1.911(1)$ & $1.906(4)$ & $1.894(3)$ \\
$\mathrm{Fe}(1)-\mathrm{N}(2)$ & $1.913(8)$ & $1.920(1)$ & $1.924(1)$ & $1.934(4)$ & - \\
$\mathrm{Fe}(2)-\mathrm{N}(1)$ & $1.917(7)$ & $1.909(1)$ & $1.904(1)$ & $1.919(4)$ & - \\
$\mathrm{Fe}(2)-\mathrm{N}(2)$ & $1.923(7)$ & $1.919(1)$ & $1.932(1)$ & $1.937(4)$ & $1.945(2)$ \\
$\mathrm{N}(1)-\mathrm{Fe}(1)-\mathrm{Fe}(2)$ & $49.2(2)$ & $47.69(5)$ & $46.90(4)$ & $48.23(12)$ & $70.11(8)$ \\
$\mathrm{N}(2)-\mathrm{Fe}(1)-\mathrm{Fe}(2)$ & $49.3(2)$ & $47.94(4)$ & $47.72(4)$ & $48.69(11)$ & - \\
$\mathrm{N}(1)-\mathrm{Fe}(2)-\mathrm{Fe}(1)$ & $48.4(2)$ & $47.54(5)$ & $47.12(4)$ & $47.80(12)$ & - \\
$\mathrm{N}(2)-\mathrm{Fe}(2)-\mathrm{Fe}(1)$ & $48.9(2)$ & $47.98(4)$ & $47.46(4)$ & $48.58(12)$ & $69.94(7)$ \\
\hline
\end{tabular}

2.3 双膦配体取代产物 $\left[\mathrm{Fe}_{2}\left(\mathrm{~N}_{2} \mathrm{C}_{5} \mathrm{H}_{10}\right)(\mathrm{CO})_{4}(\mu\right.$-dppe)] $3 \mathbf{a}$ 和 $\left[\mathrm{Fe}_{2}\left(\mathrm{~N}_{2} \mathrm{C}_{5} \mathrm{H}_{10}\right)(\mathrm{CO})_{5}\left(\kappa^{2}-\mathrm{dppbz}\right)\right] \mathbf{3 b}$ 的合成及表征 我们发现, 当使用上述合成 $2 \mathbf{a}$ 和 $\mathbf{2 b}$ 的方法进行双
齿膦配体化合物的合成时，产率较低. 用红外光谱监测 反应，发现有多种产物(或中间体)生成. 当采用光照方 法进行羰基的取代反应时，能以中等收率分离得到目标 产物. 如图式 1 所示, 化合物 $\mathbf{1}$ 和双齿膦配体溶解于甲 苯溶液中, 采用 $365 \mathrm{~nm}$ 的 LED 灯在室温条件下光照反 应溶液. 利用红外光谱跟踪反应进程, 反应历经 $5 \mathrm{~h}$ 完 成, 减压蒸出溶剂, 利用二氯甲烷/正己烷重结晶, 分别 以 $53 \%$ 和 $46 \%$ 的分离产率得到化合物 $\mathbf{3 a}$ 和 $\mathbf{3 b}$.

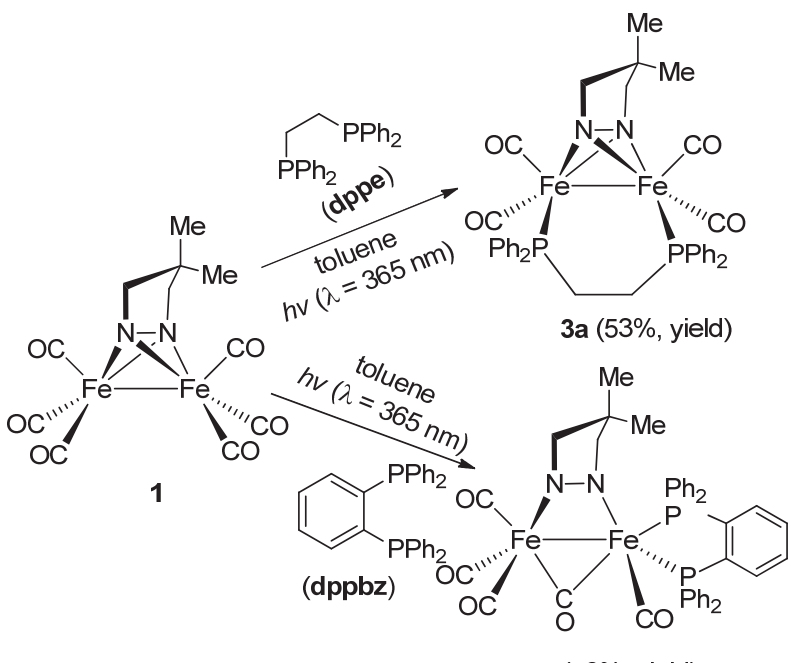

3b $(46 \%$, yield $)$

图式 1 化合物 $3 \mathrm{a}$ 和 $3 \mathrm{~b}$ 的合成

Scheme 1 The synthesis of compounds $\mathbf{3 a}$ and $\mathbf{3 b}$

化合物 3a 的 ${ }^{31} \mathrm{P}$ NMR 谱图呈现一个单峰, 化学位 移位于 $\delta 95 . \mathbf{3 b}$ 中的两个 $\mathrm{P}$ 虽处于不同的化学环境, 但 两个 $\mathrm{P}$ 螯合在一个 $\mathrm{Fe}$ 上时, 会发生快速 “旋转”, 交换 位置, 从而在核磁共振谱上难以区分 ${ }^{[28]}$. 因此, 3b 的 ${ }^{31} \mathrm{P}$ NMR 图也呈现单峰, 化学位移为 $\delta$ 93. 红外光谱上, 3a 的羰基的伸缩振动 $v_{\mathrm{CO}}$ 为 $1984,1940,1925$ 和 $1900 \mathrm{~cm}^{-1}$; $3 \mathbf{b}$ 的 $v_{\mathrm{CO}}$ 和 3a 相近, 吸收带位于 1990, 1947, 1919 和 $1895 \mathrm{~cm}^{-1}$; 相比于单齿膦配体化合物 $\mathbf{2 a}$ 和 $\mathbf{2 b}, v_{\mathrm{CO}}$ 平均 向低波数移动约 $30 \mathrm{~cm}^{-1}$, 如图 6 .

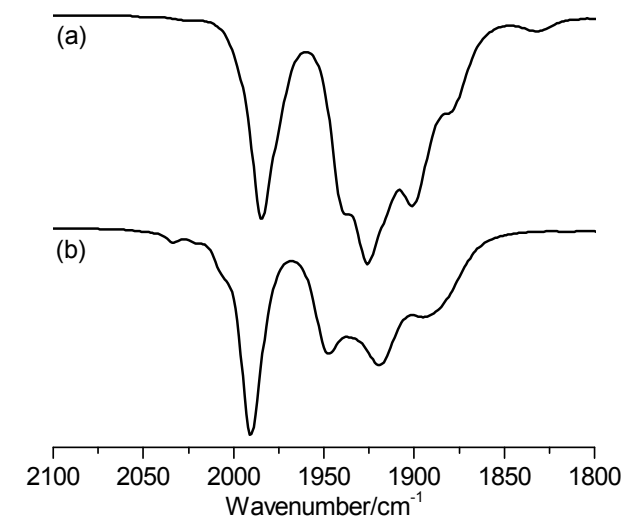

图 6 化合物 3a (a) 和 $3 b(b)$ 的红外光谱图

Figure 6 IR spectra of $\mathbf{3 a}(\mathrm{a})$ and $\mathbf{3 b}$ (b) in $v_{\mathrm{CO}}$ region 
我们利用 $X$ 射线晶体衍射分别对化合物 $3 \mathbf{a}$ 和 $\mathbf{3 b}$ 进行了结构表征, 二者结构有很大的差异, 如图 7 所示. 化合物 $3 \mathbf{a}$ 具有与 $\mathbf{1}, \mathbf{2 a}$ 和 $\mathbf{2 b}$ 相似的 $\mathrm{Fe}_{2} \mathrm{~N}_{2}$ 蝶形结构. dppe 与 $\mathrm{Fe}(\mathrm{I}) \mathrm{Fe}(\mathrm{I})$ 中心桥联配位, 处于 $\mathrm{Fe}(\mathrm{I}) \mathrm{Fe}(\mathrm{I})$ 底部的 顺式位置, 形成扭曲的 $(\mathrm{Fe}-\mathrm{P}-\mathrm{C})_{2}$ 六元环, 与文献报道的 $\mathrm{Fe}_{2}\left(\mathrm{SC}_{3} \mathrm{H}_{6} \mathrm{~S}\right)(\mathrm{CO})_{4}(\mu \text {-dppe })^{[29]}$ 结构类似. 3a 中 $\mathrm{Fe}-\mathrm{Fe}$ 键 长为 2.558(1) $\AA$, 处于 $\mathbf{2 a}$ 和 $\mathbf{2 b}$ 的 $\mathrm{Fe}-\mathrm{Fe}$ 键长之间, 其 它的键长和键角数值与 $\mathbf{2 a}$ 和 $\mathbf{2 b}$ 相比稍有变化.
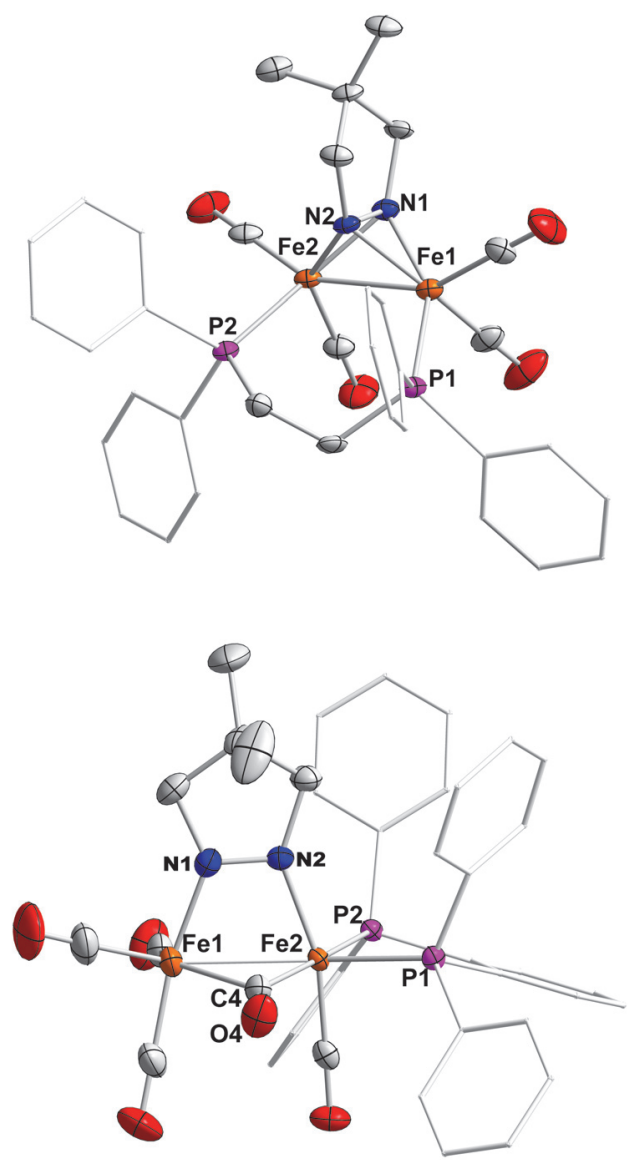

图 7 化合物 $\mathbf{3 a}$ 和 $\mathbf{3 b}$ 的晶体结构

Figure 7 Molecular structure (50\% probability thermal ellipsoids) of compounds $\mathbf{3 a}$ and $\mathbf{3 b}$. For clarity, the phenyl groups, cyclohexyl groups and hydrogen atoms are omitted

化合物 3b 与上述 $\mathrm{Fe}_{2}\left(\mathrm{~N}_{2} \mathrm{C}_{5} \mathrm{H}_{10}\right)(\mathrm{CO})_{6-x}\left(\mathrm{PR}_{3}\right)_{x}$ 类化合 物的结构完全不同, 分子中 $\mathrm{Fe}_{2} \mathrm{~N}_{2}$ 蝶形结构打开, 成平 面四边形. 更为有趣的是, $\mathrm{Fe}(\mathrm{CO})_{3}$ 单元上只有一个 $\mathrm{CO}$ 被取代, 且 $d p p b z$ 螯合 $\mathrm{Fe}$ 中心, 形成 $\mathrm{Fe}(\mathrm{CO})_{2}(\mathrm{dppv})$; 其 中一个 $\mathrm{CO}$ 又与 $\mathrm{Fe}(\mathrm{I}) \mathrm{Fe}(\mathrm{I})$ 桥连, 呈现 $\mathrm{Fe}-\mathrm{CO}-\mathrm{Fe}$ 的 “旋 转” 构型. 其中, $\mathrm{Fe}-\mathrm{Fe}$ 键长为 2.5881(6) $\AA$, 比 3a 中 $\mathrm{Fe}-\mathrm{Fe}$ 键长 $0.03 \AA$. 迄今为止, 文献只报道了两例具有 “旋转” 构型的 $\mathrm{Fe}_{2}(\mathrm{SR})_{2}(\mathrm{CO})_{4}(\mathrm{P}-\mathrm{P})$ 化合物 ${ }^{[23,24]}, \mathrm{Fe}-\mathrm{Fe}$ 键长分别为 2.662(2) $\AA$ 和 2.5434(4) $\AA$; 3b 的 $\mathrm{Fe}-\mathrm{Fe}$ 键 长介于二者之间. 桥连羰基的 $\mathrm{C}$ 原子到两个 $\mathrm{Fe}$ 原子之 间距离不相等, $\mathrm{Fe}(1)-\mathrm{C}(4)$ 为 2.063(3) $\AA$ 比 $\mathrm{Fe}(2)-\mathrm{C}(4)$ $1.939(3) \AA$ 长 $0.12 \AA$. 另外, 两个 $\mathrm{Fe}$ 中心都呈扭曲的八
面体构型， $\mathrm{P}(1)-\mathrm{Fe}(2)-\mathrm{Fe}(1)$ 的键角为 $151.35(3)^{\circ}$, $\mathrm{C}(4)-\mathrm{Fe}(2)-\mathrm{P}(2)$ 为 $170.86(10)^{\circ} ; \mathrm{N}(2)-\mathrm{Fe}(2)-\mathrm{Fe}(1)$ 的 键角为 $69.94(7)^{\circ}$ 远远小于 $\mathrm{N}(2)-\mathrm{Fe}(2)-\mathrm{P}(1)$ 的 $102.99(7)^{\circ}$. 平面 $\mathrm{N}(1)-\mathrm{Fe}(1)-\mathrm{Fe}(2)-\mathrm{N}(2)$ 并非完全垂 直于平面 $\mathrm{Fe}(1)-\mathrm{C}(4)-\mathrm{Fe}(2)$, 其二面角为 79.4(2) ${ }^{\circ}$. 尽 管化合物 $3 \mathbf{b}$ 不具有 $\mathrm{Fe}_{2} \mathrm{~N}_{2}$ 蝶形结构, 但存在 $\mathrm{Fe}-\mathrm{CO}-$ $\mathrm{Fe}$ 结构单元, 能为探索合成 $[\mathrm{FeFe}]$ 氢化酶活性中心结构 提供新的途径和思路.

\section{3 结论}

化合物 1 具有与 $\mathrm{Fe}_{2}(\mathrm{SR})_{2}(\mathrm{CO})_{6}$ 相似的结构, 其中的 $\mathrm{CO}$ 配体可以被膦配体取代, 生成一系列有趣的 $\mathrm{Fe}_{2}\left(\mathrm{~N}_{2} \mathrm{C}_{5} \mathrm{H}_{10}\right)(\mathrm{CO})_{6-x} \mathrm{~L}_{x}$ 衍生物. 1 和双齿膦配体 dppe 反应 时, 取代反应发生在两个 $\mathrm{Fe}$ 上, 生成对称取代产物 $\mathbf{3 a}$; 与刚性膦配体 $\mathrm{dppbz}$ 反应时, 取代反应发生在一个 $\mathrm{Fe}$ 上，生成的化合物 $\mathbf{3 b}$ 具有独特的 $\mathrm{Fe}-\mathrm{CO}-\mathrm{Fe}$ 的 “旋转” 构型, 可视为 $[\mathrm{FeFe}]$ 氢化酶活性中心还原态的模型化合 物. 我们后续的研究将探索这类化合物催化 $\mathrm{H}^{+}$还原制 $\mathrm{H}_{2}$ 的性质，并且通过改变配体的种类调节金属铁中心 的氧化还原电位, 优化其催化性能.

\section{4 实验部分}

实验过程中所有的合成都在 $\mathrm{N}_{2}$ 氛围下, 采用标准 的 Schlenk 操作方法. 配体 4,4-二甲基-4,5-二氢-3H-吡咯 参照文献 ${ }^{[30]}$ 合成. 如无特别说明, 其它化学药品和试剂 均通过正常商业渠道购买.

\section{$4.1 \mathrm{Fe}_{2}\left(\mathrm{~N}_{2} \mathrm{C}_{5} \mathrm{H}_{10}\right)(\mathrm{CO})_{6} 1$ 的合成}

氮气氛围下，向十二羰基三铁 $(3.00 \mathrm{~g}, 6.0 \mathrm{mmol})$ 的 甲苯 $(100 \mathrm{~mL})$ 溶液中, 加入 4,4-二甲基-4,5-二氢-3H-吡 咯(0.62 g, $6.3 \mathrm{mmol})$, 搅拌回流, 溶液由墨绿色逐渐变 为深褐色. 利用红外光谱跟踪反应进程, $2 \mathrm{~h}$ 后停止反 应. 减压蒸除溶剂, 正己烷溶解产物, 硅胶柱柱层析, 以石油醚为洗脱剂, 分离得到 $1.54 \mathrm{~g}$ 橙色固体, 产率 68\%. ${ }^{1} \mathrm{H}$ NMR (400 MHz, $\left.\mathrm{CD}_{2} \mathrm{Cl}_{2}\right) \delta: 3.41\left(4 \mathrm{H}, 2 \mathrm{CH}_{2}\right)$, $1.32\left(6 \mathrm{H}, 2 \mathrm{CH}_{3}\right)$; FT-IR $\left(\mathrm{CH}_{2} \mathrm{Cl}_{2}\right) v_{\mathrm{CO}}: 2069,2022,1986$ $\mathrm{cm}^{-1}$. Anal. calcd for $\mathrm{C}_{11} \mathrm{H}_{10} \mathrm{Fe}_{2} \mathrm{~N}_{2} \mathrm{O}_{6}$ : C 34.96, $\mathrm{H} 2.67, \mathrm{~N}$ 7.41; found C 35.07, H 2.73, N 7.53.

\section{$4.2 \mathrm{Fe}_{2}\left(\mathrm{~N}_{2} \mathrm{C}_{5} \mathrm{H}_{10}\right)(\mathrm{CO})_{5} \mathrm{PPh}_{3} 2 \mathrm{a}$ 的合成}

氮气氛围下, 向 $1(0.38 \mathrm{~g}, 1 \mathrm{mmol})$ 的甲苯 $(80 \mathrm{~mL})$ 溶 液中, 加入 $0.075 \mathrm{~g} \mathrm{Me}_{3} \mathrm{NO}(1 \mathrm{mmol})$ 的乙腈 $(15 \mathrm{~mL})$ 溶液. 搅拌反应 $15 \mathrm{~min}$ 后, 加入三苯基膦 $(0.26 \mathrm{~g}, 1 \mathrm{mmol})$ 固 体, 溶液由亮橙色变为深褐色. 室温下搅拌 $3 \mathrm{~h}$, 停止反 应, 减压蒸出溶剂, 用二氯甲烷溶解, 硅藻土过滤. 浓 缩滤液, 正己烷沉淀洗涤, 过滤干燥得 $0.50 \mathrm{~g}$ 棕色固体. ${ }^{1} \mathrm{H}$ NMR (400 MHz, $\mathrm{CD}_{2} \mathrm{Cl}_{2}$ ) $\delta: 7.33 \sim 7.46$ (15H, 3ArH), $2.91 \sim 3.03\left(4 \mathrm{H}, 2 \mathbf{C H}_{2}\right), 0.90 \sim 1.48\left(6 \mathrm{H}, 2 \mathbf{C H}_{3}\right) ;{ }^{31} \mathrm{P} \mathrm{NMR}$ $\left(400 \mathrm{MHz}, \mathrm{CD}_{2} \mathrm{Cl}_{2}\right) \delta$ : 67; FT-IR $\left(\mathrm{CH}_{2} \mathrm{Cl}_{2}\right) v_{\mathrm{CO}}: 2055$, 
1981, 1956, $1904 \mathrm{~cm}^{-1}$. Anal. calcd for $\mathrm{C}_{28} \mathrm{H}_{25} \mathrm{Fe}_{2} \mathrm{~N}_{2} \mathrm{O}_{5} \mathrm{P}$ : C 54.49, H 4.90, N 4.54; found C 54.61, H 4.63, N 4.64.

\section{$4.3 \mathrm{Fe}_{2}\left(\mathrm{~N}_{2} \mathrm{C}_{5} \mathrm{H}_{10}\right)(\mathrm{CO})_{5} \mathrm{PCy}_{3} 2 \mathrm{~b}$ 的合成}

化合物 $\mathbf{2 b}$ 的合成方法与 $\mathbf{2 a}$ 相同. ${ }^{1} \mathrm{H}$ NMR (400 $\left.\mathrm{MHz}, \mathrm{CD}_{2} \mathrm{Cl}_{2}\right) \delta: 3.47\left(4 \mathrm{H}, 2 \mathrm{NCH}_{2}\right), 1.38 \sim 2.02(39 \mathrm{H}$, $\left.2 \mathrm{CH}_{3}, \mathrm{PCy}_{3}\right) .{ }^{31} \mathrm{P}$ NMR (400 MHz, $\left.\mathrm{CD}_{2} \mathrm{Cl}_{2}\right) \delta: 70$. FT-IR $\left(\mathrm{CH}_{2} \mathrm{Cl}_{2}, v_{\mathrm{CO}}\right): 2031,1968,1950,1906 \mathrm{~cm}^{-1}$. Anal. calcd for $\mathrm{C}_{28} \mathrm{H}_{43} \mathrm{Fe}_{2} \mathrm{~N}_{2} \mathrm{O}_{5} \mathrm{P}$ : C 54.49, $\mathrm{H}$ 4.90, $\mathrm{N}$ 4.54; found $\mathrm{C}$ 54.61, H 4.63, N 4.64.

\section{$4.4\left[\mathrm{Fe}_{2}\left(\mathrm{~N}_{2} \mathrm{C}_{5} \mathrm{H}_{10}\right)(\mathrm{CO})_{5}(\mu\right.$-dppe $\left.)\right] 3 \mathrm{a}$ 的合成}

将 $0.38 \mathrm{~g}$ 化合物 $2(1 \mathrm{mmol})$ 和 $0.40 \mathrm{~g}$ 配体 dppe 溶于 $80 \mathrm{~mL}$ 甲苯, 氮气条件下光照 $(\lambda=365 \mathrm{~nm})$ 该反应体系. 用红外光谱跟踪反应进程, 约 $5 \mathrm{~h}$ 后, 反应完成. 浓缩反 应溶液, 加入正己烷沉淀, 过滤干燥得 $0.38 \mathrm{~g}$ 棕黑色固

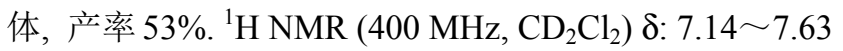
$(20 \mathrm{H}, 4 \mathrm{ArH}), 3.21 \sim 3.45,1.14 \sim 1.43\left(4 \mathrm{H}, 2 \mathrm{NCH}_{2}\right)$, $2.28 \sim 2.49(4 \mathrm{H}, 2 \mathrm{PCH}), 0.89\left(6 \mathrm{H}, 2 \mathbf{C H}_{3}\right) ;{ }^{31} \mathrm{P}$ NMR $(400$ $\left.\mathrm{MHz}, \mathrm{CD}_{2} \mathrm{Cl}_{2}\right) \delta$ : 95. FT-IR $\left(\mathrm{CH}_{2} \mathrm{Cl}_{2}, v_{\mathrm{CO}}\right)$ : 1984, 1940 , 1925 和 $1900 \mathrm{~cm}^{-1}$. Anal. calcd for $\mathrm{C}_{35} \mathrm{H}_{34} \mathrm{Fe}_{2} \mathrm{~N}_{2} \mathrm{O}_{4} \mathrm{P}_{2}: \mathrm{C}$ 58.36, H 4.76, N 3.89; found C 58.41, H 4.78, N 3.92.

\section{$4.5\left[\mathrm{Fe}_{2}\left(\mathrm{~N}_{2} \mathrm{C}_{5} \mathrm{H}_{10}\right)(\mathrm{CO})_{5}\left(K^{2}-\mathrm{dppbz}\right)\right] 3 \mathrm{~b}$ 的合成}

化合物 3b 的合成方法与 3a 相同. ${ }^{1} \mathrm{H}$ NMR (400 $\left.\mathrm{MHz}, \mathrm{CD}_{2} \mathrm{Cl}_{2}\right) \delta: 7.22 \sim 7.60(24 \mathrm{H}, 5 \mathrm{ArH}), 3.45 \sim 1.15$ $\left(4 \mathrm{H}, 2 \mathrm{NCH}_{2}\right), 0.47\left(6 \mathrm{H}, 2 \mathrm{CH}_{3}\right) ;{ }^{31} \mathrm{P} \mathrm{NMR}(400 \mathrm{MHz}$, $\left.\mathrm{CD}_{2} \mathrm{Cl}_{2}\right) \delta$ : 93; FT-IR $\left(\mathrm{CH}_{2} \mathrm{Cl}_{2}\right) v_{\mathrm{CO}}$ : 1990, 1947, 1919, $1895 \mathrm{~cm}^{-1}$. Anal. calcd for $\mathrm{C}_{40} \mathrm{H}_{34} \mathrm{Fe}_{2} \mathrm{~N}_{2} \mathrm{O}_{5} \mathrm{P}_{2}$ : C 60.33, $\mathrm{H}$ 4.30, N 3.52; found C 60.43, H 4.42, N 3.57.

\section{6 晶体结构的测定与解析}

$\mathrm{X}$ 射线晶体结构分析在 Bruker SMART APEX II $\mathrm{CCD}$ 衍射仪上收集数据, 采用石墨单色化的 Mo K $\alpha$ 射 线 $(\lambda=0.0707 \AA)$. 所收集的数据通过经验吸收校正, 结 构由直接法解出. 采用 SHELXTL-97 软件进行结构解析 和精修. CCDC: 1494954 (1), 1494955 (2a), 1494956 (2b), 1494966 (3a) 和 1494957 (3b).

\section{致谢}

感谢孙頔博士在 $X$ 射线单晶结构衍射测试和数据 解析等方面提供的帮助和建议.

\section{References}

[1] (a) Lubitz, W.; Reijerse, E.; van Gastel, M. Chem. Rev. 2007, 107, 4331; (b) Ginovska-Pangovska, B.; Ho, M.-H.; Linehan, J. C.; Cheng, Y.; Dupuis, M.; Raugei, S.; Shaw, W. J. Biochim. Biophys. Acta. Bioenerg. 2014, 1837, 131; (c) Frey, M. ChemBioChem 2002, 3, 153; (d) Silakov, A.; Kamp, C.; Reijerse, E.; Happe, T.; Lubitz, W. Biochemistry 2009, 48, 7780; (e) Capon, J.-F.; Gloaguen, F.; Pétillon, F. Y.; Schollhammer, P.; Talarmin, J. Coord. Chem. Rev. 2009, 253, 1476; (f) Fontecilla-Camps, J. C.; Volbeda, A.; Cavazza, C.; Nicolet, Y. Chem. Rev. 2007, 107, 4273.

[2] (a) Siegbahn, P. E. M.; Tye, J. W.; Hall, M. B. Chem. Rev. 2007, 107,
4414. (b) Berggren, G.; Adamska, A.; Lambertz, C.; Simmons, T. R.; Esselborn, J.; Atta, M.; Gambarelli, S.; Mouesca, J.-M.; Reijerse, E.; Lubitz, W.; Happe, T.; Artero, V.; Fontecave, M. Nature 2013, 499, 66.

[3] (a) Nicolet, Y.; Piras, C.; Legrand, P. C.; Hatchikian E.; FontecillaCamps, J. C. Structure 1999, 7, 13. (b) Peters, J. W.; Lanzilotta, W. N.; Lemon, B. J.; Seefeldt, L. C. Science 1998, 282, 1853. (c) Pandey, A. S.; Harris, T. V.; Giles, L. J.; Peters, J. W.; Szilagyi, R. K. J. Am. Chem. Soc. 2008, 130, 4533.

[4] Adams, M. W. W. J. Biol. Chem. 1987, 262, 15054.

[5] Kubas, G. J. Chem. Rev. 2007, 107, 4152.

[6] Knörzer, P.; Silakov, A.; Foster, C. E.; Armstrong, F. A.; Lubitz, W.; Happe, T. J. Biol. Chem. 2012, 287, 1489.

[7] Simmons, T. R.; Berggren, G.; Bacchi, M.; Fontecave, M.; Artero, V. Coord. Chem. Rev. 2014, 270-271, 127.

[8] Chernev, P.; Lambertz, C.; Brünje, A.; Leidel, N.; Sigfridsson, K. G. V.; Kositzki, R.; Hsieh, C.-H.; Yao, S.; Schiwon, R.; Driess, M.; Limberg, C.; Happe, T.; Haumann, M. Inorg. Chem. 2014, 53, 12164.

[9] Tschierlei, S.; Ott, S.; Lomoth, R. Energy Environ. Sci. 2011, 4, 2340.

[10] (a) Zhao, X.; Georgakaki, I. P.; Miller, M. L.; Yarbrough, J. C.; Darensbourg, M. Y. J. Am. Chem. Soc. 2001, 123, 9710. (b) Song, L.-C.; Yang, Z.-Y.; Bian, H.-Z.; Liu, Y.; Wang, H.-T.; Liu, X.-F.; Hu, Q.-M. Organometallics 2005, 24, 6126. (c) Song, L.-C.; Liu, X.-F.; Ming, J.-B.; Ge, J.-H.; Xie, Z.-J.; Hu, Q.-M. Organometallics 2010 , 29, 610. (d) Song, L. C. Acc. Chem. Res. 2005, 38, 21. (e) Gao, W. M.; Liu, J. H.; Akermark, B.; Sun, L. C. Inorg. Chem. 2006, 45, 9169 .

[11] (a) Dong, W.; Wang, M.; Liu, X.; Jin, K.; Li, G.; Wang, F.; Sun, L. Chem. Commun. 2006, 305. (b) Wang, N.; Wang, M.; Zhang, T.; Li, P.; Liu, J.; Sun, L. Chem. Commun. 2008, 5800. (c) Ezzaher, S.; Capon, J.-F.; Gloaguen, F.; Pétillon, F. Y.; Schollhammer, P.; Talarmin, J. Inorg. Chem. 2009, 48, 2; (d) Li, P.; Wang, M.; Chen, L.; Liu, J.; Zhao, Z.; Sun, L. Dalton Trans. 2009, 1919. (e) Schwartz, L.; Eilers, G.; Eriksson, L.; Gogoll, A.; Lomoth, R.; Ott, S. Chem. Commun. 2006, 520. (f) Eilers, G.; Schwartz, L.; Stein, M.; Zampella, G.; de Gioia, L.; Ott, S.; Lomoth, R. Chem. Eur. J. 2007, 13, 7075.

[12] (a) Lyon, E. J.; Georgakaki, I. P.; Reibenspies, J. H.; Darensbourg, M. Angew. Chem., Int. Ed. 1999, 38, 3178. (b) Schmidt, M.; Contakes, S. M.; Rauchfuss, T. B. J. Am. Chem. Soc. 1999, 121, 9736.

[13] (a) Capon, J.-F.; El Hassnaoui, S.; Gloaguen, F.; Schollhammer, P.; Talarmin, J. Organometallics 2005, 24, 2020. (b) Morvan, D.; Capon, J.-F.; Gloaguen, F.; Le Goff, A.; Marchivie, M.; Michaud, F.; Schollhammer, P.; Talarmin, J.; Yaouanc, J.-J.; Pichon, R.; Kervarec, N. Organometallics 2007, 26, 2042. (c) Tye, J. W.; Lee, J.; Wang, H.-W.; Mejia-Rodriguez, R.; Reibenspies, J. H.; Hall, M. B.; Darensbourg, M. Y. Inorg. Chem. 2005, 44, 5550.

[14] (a) Schilter, D.; Camara, J. M.; Huynh, M. T.; Hammes-Schiffer, S.; Rauchfuss, T. B. Chem. Rev. 2016, doi: 10. 1021/acs. chemrev. 6b00180. (b) Rauchfuss, T. B. Acc. Chem. Res. 2015, 48, 2107. (c) Lubitz, W.; Ogata, H.; Rüdiger, O.; Reijerse, E. Chem. Rev. 2014, 114, 4081. (d) Wang, N.; Wang, M.; Chen, L.; Sun, L. C. Dalton Trans. 2013, 42, 12059. (e) Wang, M.; Sun, L. ChemSusChem 2010, 3, 551.

[15] (a) Tard, C. D.; Pickett, C. J. Chem. Rev. 2009, 109, 2245. (b) McGlynn, S. E.; Mulder, D. W.; Shepard, E. M.; Broderick, J. B.; Peters, J. W. Dalton Trans. 2009, 4274. (c) Gloaguen, F.; Rauchfuss, T. B. Chem. Soc. Rev. 2009, 38, 100. (d) De Lacey, A. L.; Fernández, V. M.; Rousset, M.; Cammack, R. Chem. Rev. 2007, 107, 4304.

[16] (a) Wu, L.-Z.; Chen, B.; Li, Z.-J.; Tung, C.-H Acc. Chem. Res. 2014, 47, 2177. (b) Wang, F.; Wang, W.-G.; Wang, H.-Y.; Si, G.; Tung, C.-H.; Wu, L.-Z. ACS Catal. 2012, 407. (c) Wang, F.; Liang, W.-J.; Wang, W.-G.; Chen, B.; Feng, K.; Zhang, L.-P.; Tung, C.-H.; Wu, L.-Z. Acta Chim. Sinica 2012，70，2306. (王锋, 梁文静, 王文光, 陈涁, 冯科, 张丽萍, 佟振合, 吴骊珠, 化学学报, 2012, 70, 2306).

[17] Liu, T.; Darensbourg, M. Y. J. Am. Chem. Soc. 2007, 129, 7008.

[18] Justice, A. K.; Rauchfuss, T. B.; Wilson, S. R. Angew. Chem., Int. Ed. 2007, 46, 6152 .

[19] (a) Wang, N.; Wang, M.; Wang, Y.; Zheng, D.; Han, H.; Ahlquist, M. S. G.; Sun, L. J. Am. Chem. Soc. 2013, 135, 13688. (b) Mulder, D. W.; Ratzloff, M. W.; Shepard, E. M.; Byer, A. S.; Noone, S. M.; Peters, J. W.; Broderick, J. B.; King, P. W. J. Am. Chem. Soc. 2013 135, 6921. (c) Camara, J. M.; Rauchfuss, T. B. Nat. Chem. 2012, 4 , 26. (d) Camara, J. M.; Rauchfuss, T. B. J. Am. Chem. Soc. 2011, 133,8098 . 
[20] (a) Barton, B. E.; Zampella, G.; Justice, A. K.; De Gioia, L.; Rauchfuss, T. B.; Wilson, S. R. Dalton Trans. 2010, 39, 3011. (b) Carroll, M. E.; Barton, B. E.; Rauchfuss, T. B.; Carroll, P. J. J. Am. Chem. Soc. 2012, 134, 1884.

[21] Barton, B. E.; Rauchfuss, T. B. Inorg. Chem. 2008, 47, 2261.

[22] Tye, J. W.; Darensbourg, M. Y.; Hall, M. B. Inorg. Chem. 2006, 45, 1552.

[23] Wang, W.; Rauchfuss, T. B.; Moore, C. E.; Rheingold, A. L.; De Gioia, L.; Zampella, G. Chem. Eur. J. 2013, 19, 15476.

[24] Munery, S.; Capon, J.-F.; De Gioia, L.; Elleouet, C.; Greco, C.; Pétillon, F. Y.; Schollhammer, P.; Talarmin, J.; Zampella, G. Chem. Eur. J. 2013, 19, 15458.

[25] (a) Harb, M. K.; Apfel, U.-P.; Kübel, J.; Görls, H.; Felton, G. A. N.; Sakamoto, T.; Evans, D. H.; Glass, R. S.; Lichtenberger, D. L.; El-khateeb, M.; Weigand, W. Organometallics 2009, 28, 6666. (b) Harb, M. K.; Niksch, T.; Windhager, J.; Görls, H.; Holze, R.; Lockett, L. T.; Okumura, N.; Evans, D. H.; Glass, R. S.; Lichtenberger, D. L.; El-khateeb, M.; Weigand, W. Organometallics 2009, 28, 1039. (c) Harb, M. K.; Windhager, J.; Daraosheh, A.; Gorls, H.; Lockett, L. T.; Okumura, N.; Evans, D. H.; Glass, R. S.; Lichtenberger, D. L.;
El-Khateeb, M.; Weigand, W. Eur. J. Inorg. Chem. 2009, 3414.

[26] (a) Das, P.; Capon, J.-F.; Gloaguen, F.; Pétillon, F. Y.; Schollhammer, P.; Talarmin, J.; Muir, K. W. Inorg. Chem. 2004, 43, 8203. (b) Zaffaroni, R.; Rauchfuss, T. B.; Fuller, A.; De Gioia, L.; Zampella, G Organometallics 2013, 32, 232. (c) Gimbert-Surinach, C.; Bhadbhade, M.; Colbran, S. B. Organometallics 2012, 31, 3480.

[27] Volkers, P.; Rauchfuss, T. B. J. Inorg. Biochem. 2007, 101, 1748.

[28] (a) Carroll, M. E.; Chen, J.; Gray, D. E.; Lansing, J. C.; Rauchfuss, T. B.; Schilter, D.; Volkers, P. I.; Wilson, S. R. Organometallics 2014, 33, 858. (b) Justice, A. K.; Zampella, G.; De Gioia, L.; Rauchfuss, T. B.; van der Vlugt, J. I.; Wilson, S. R. Inorg. Chem. 2007, 46, 1655 .

[29] Adam, F. I.; Hogarth, G.; Kabir, S. E.; Richards, I. C. R. Chim. 2008 , 11,890 .

[30] van Loevezijn, A.; Venhorst, J.; Iwema Bakker, W. I.; de Korte, C. G.; de Looff, W.; Verhoog, S.; van Wees, J.-W.; van Hoeve, M.; van de Woestijne, R. P.; van der Neut, M. A. W.; Borst, A. J. M.; van Dongen, M. J. P.; de Bruin, N. M. W. J.; Keizer, H. G.; Kruse, C. G J. Med. Chem. 2011, 54, 7030. 